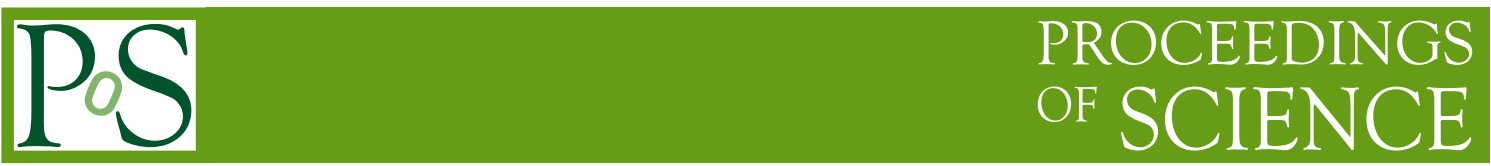

\title{
Depleted CMOS sensors for HL-LHC
}

\section{H. Pernegger ${ }^{* \dagger}$}

CERN EP Department, $\mathrm{CH}-1211$ Geneve 23

E-mail: heinz.perneggerecern.ch

\begin{abstract}
The upgrade of the ATLAS tracking detector (ITk) for the High-Luminosity Large Hadron Collider at CERN requires the development of novel radiation hard silicon sensor technologies. For the development of monolithic CMOS sensors for ATLAS we develop fully depleted CMOS monolithic active pixel sensors (DMAPS) to achieve radiation hard CMOS sensors inline with ATLAS ITk specifications. Based on initial studies on prototype sensors we have now developed, produced and tested first full-size depleted CMOS sensors in different CMOS processes along two design approaches: Designs using "small" electrodes were produced in the TowerJazz 180nm imaging process and designs using "large" electrodes were manufactured in AMS 180nm and LFoundry 150nm CMOS processes. The sensors achieve full depletion of the detection layer through a combination of high-resistivity substrates and high bias voltages. The matrix readout architectures are optimised to cope with the LHC 25ns bunch structure and the high hit-rates as expected for the outermost ITk pixel layer. The paper introduces the different design approaches and will present measurements results to assess their performance in view of future applications as radiation hard monolithic CMOS sensors for trackers.
\end{abstract}

The 27th International Workshop on Vertex Detectors - VERTEX2018

22-26 October 2018

MGM Beach Resorts, Muttukadu, Chennai, India

\footnotetext{
* Speaker.

${ }^{\dagger}$ on behalf of the ATLAS CMOS Pixel Collaboration.
} 


\section{Introduction}

The ATLAS detector [1] at the Large Hadron Collider (LHC, CERN, Geneva, Switzerland) will carry out a major upgrade to its tracking system in view of the High-Luminosity LHC phase [2]. We develop radiation hard CMOS sensors as option for the $5^{\text {th }}$ layer of the pixel barrel detector of the ITk. This encompasses the development of monolithic CMOS sensors with radiation hardness up to $1.5 \times 10^{15} \mathrm{n}_{e q} / \mathrm{cm}^{2}$, hit rates up to $2 \mathrm{MHz} / \mathrm{mm}^{2}$ and readout architecture compatible with the ATLAS data taking in HL- LHC. Due to their cost advantage, monolithic CMOS sensors are an interesting sensor technology to equip larger surfaces in future trackers. The absence of bumpbonding, necessary for hybrid pixel detectors, substantially reduces costs and assembly complexity. It furthermore allows for very thin detector layers down to $50 \mu \mathrm{m}$ thick silicon sensors which reduces the overall material budget of the tracker. Additionally the pixel pitch is not constrained by bump-bonding technology which allows in some designs for pixel pitches significantly smaller than $50 \times 50 \mu \mathrm{m}^{2}$, the current baseline pitch for pixel front-end chips of the ATLAS and CMS experiments for HL-LHC [3, 4].

\section{Sensor designs investigated for ATLAS}

In the context of the ATLAS development for radiation hard monolithic CMOS sensors we investigate two principal pixel cell designs: In the so-called "large electrode design" the collection n-well assumes a large area fraction of the pixel and signal amplification and digital circuit are contained in the charge collection n-well. In the so-called "small electrode design" the collection $\mathrm{n}$-well assumes a small area fraction of the pixel and amplification and digital circuit is placed in separate p-wells. Both design variants are shown in figure 1.

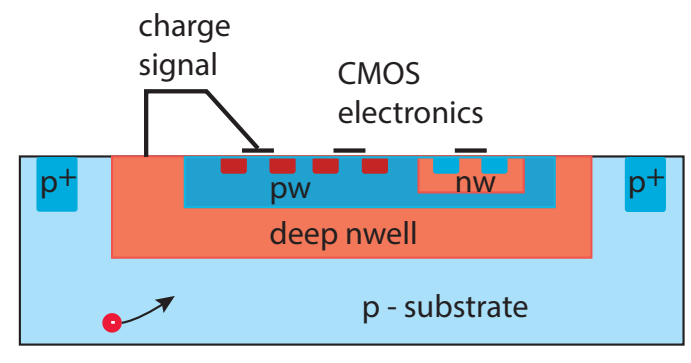

(a)

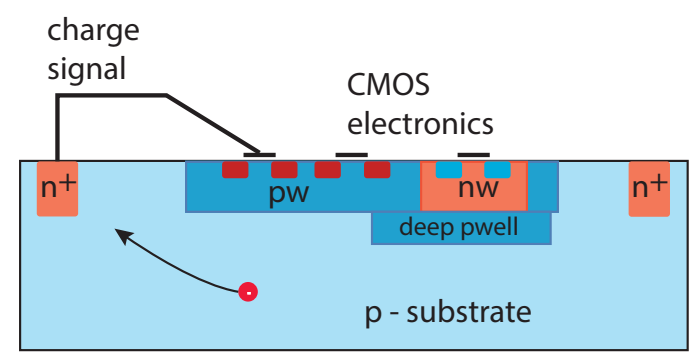

(b)

Figure 1: Two principal pixel cell designs are investigated for ATLAS monolithic sensors: (a) Large electrode design: the charge collecting deep nwell encloses the complete CMOS electronics. (b) Small electrode design: the charge collection node is placed outside the CMOS electronics area.

\subsection{Large electrode-size CMOS sensors for ATLAS}

In large-electrode designs the preamplifier circuit and digital logic are embedded in a large deep n-well, which acts as the charge collection node. The junction is formed between the collection n-well and the high resistivity p-type substrate (typically 80 to $2000 \Omega \mathrm{cm}$ in our sensors). The chosen processes (AMS $180 \mathrm{~nm}$ and LFoundry $150 \mathrm{~nm}$ ) include the possibility to apply a bias voltage in excess of $100 \mathrm{~V}$. This combination of substrate and bias voltages has been shown to allow for 
the full deletion of $300 \mu \mathrm{m}$ thick substrates and can provide a depletion width of more than $100 \mu \mathrm{m}$ even after irradiation of $10^{15} \mathrm{n}_{e q} / \mathrm{cm}^{2}$ [11].

Two different large-electrode sensors have recently been designed and tested for a possible application in the ATLAS ITk outermost pixel layer:

- The "LF-Monopix" sensor $[5,10]$ has been produced in the LFoundry $150 \mathrm{~nm}$ process and features a pixel matrix of $129 \times 36$ pixels of $50 \times 250 \mu \mathrm{m}^{2}$ pixel size on a $>2 \mathrm{k} \Omega \mathrm{cm}$ p-type substrate. Each pixel contains a charge sensitive pre-amplifier, discriminator, threshold adjustment and a 8-bit Time-Over-Threshold / Time-Of-Arrival digital logic, which is readout through a column-drain readout architecture. In addition to the pixel address of a hit, the leading-edge and trailing edge time stamps are also asserted to the column bus once the pixel is clocked, as the time stamps are recorded locally in pixel. The sensor is thinned and is reverse biased through the backside.

- The "ATLASPix1'Simple' sensor [12] has been produced in the AMS aH18 180nm process and features a pixel matrix of $400 \times 25$ pixels of $40 \times 130 \mu \mathrm{m}^{2}$ pixel size on 80 and $200 \Omega \mathrm{cm}$ p-type substrate. The pixel contains a charge sensitive amplifier, 4-bit threshold adjustment and discriminator. The signal output of each pixel is routed via an individual line to the periphery, where the Time-Of-Arrival and Time-Over-Threshold is measured. Triggered and trigger-less readout architectures have been implemented for this sensor type. Results in this paper refer to the trigger-less readout of the matrix.

The total input capacitance to the amplifier, represented by the n-well capacitance to all boundaries plus the capacitance between the deep p-well and the deep n-well, is large (up to several $100 \mathrm{fF}$, depending on layout), influencing the noise, power, and timing performance [8]. In addition, potential capacitive coupling of digital transient signals into the sensor must be prevented by dedicated design. The main advantage of large-electrode designs is however the already demonstrated radiation hardness. The average drift path of ionisation charge is along a more uniform strong electric field in a thick substrate. Due to the large ionisation charge deposited in the thick depletion layer they exhit a larger signal amplitude and high detection efficiency even with the radiation-induced traps after irradiation.

\subsection{Small electrode-size CMOS sensors for ATLAS}

Small collection electrode CMOS sensors implement a small collection diode sitting outside of the deep p-well where all the electronics is implemented. One of the advantages is that the analog circuitry requires only a small fraction of the pixel. Hence a comparably large fraction of the pixel surface is available for digital circuitry. Due to small surface needs for the analog parts, generally smaller pixel pitches can be obtained while still featuring the required complex digital in-pixel electronics. Another advantage of the small electrode design is that analog circuitry and digital logic can be separated, which results in superior electrical isolation between digital signals and analog front-end to reduce cross-talk. Present designs include the full analog front-end and digital logic for asynchronous or synchronous readout in pixel pitches of approximately $36 \mu \mathrm{m}$ to $40 \mu \mathrm{m}$ pitch. The small collection electrode (typically $3 \mu \mathrm{m}$ diameter, surrounded by a spacing of $3-4 \mu \mathrm{m}$ to the nearby $\mathrm{p}$-well, leads to a very small capacitance of typically $\approx 2 \mathrm{fF}$, which enables low 
ENC noise of less than $20 \mathrm{e}^{-}$and analog power dissipation of only $75 \mathrm{~mW} / \mathrm{cm}^{2}$ in our designs. A possible disadvantage of this design lies in the non-uniform field with a field minimum at the pixel corner, which can result in inefficiency after irradiation.

Two different readout architectures have been designed and implemented for small electrode CMOS sensors on the "STREAM1" reticle in the TowerJazz 180nm process by using a process modification for improved radiation hardness on $25 \mu \mathrm{m}$ epitaxial p-type substrate $[6,7]$ :

- Asynchronous readout scheme ("MALTA" sensor with $36 \times 36 \mu \mathrm{m}^{2}$ pixel pitch and $512 \times 512$ pixel matrix) [9]: The asynchronous design avoids the propagation of the clock in the active pixel matrix region, which further reduce risks of cross talk and provides an optimal lowpower operation. The address, timing and charge information for each hit is encoded in the asynchronous signals propagated through a column bus to the periphery where the encoding of the pixel address and the measurement of the time of arrival of asynchronous signals coming from the matrix is done. The encoded information is then buffered and transmitted off the CMOS sensors.

- Synchronous readout scheme ("TJ-Monopix" sensor with $36 \times 40 \mu \mathrm{m}^{2}$ pixel pitch and $224 \times 448$ pixel matrix) [10] comparable to the LF-Monopix described above.

\section{Recent results of sensor qualifications}

Recent effort has been focused to qualify the different sensors for use in the ITk outermost layer according to its specifications. Among the most essential requirements are an efficiency in excess of $97 \%$ after full lifetime irradiation and meeting the stringent requirements for signals being timetagged within $25 \mathrm{~ns}$ so that they are associated with the correct bunch-crossing of LHC. Sensors are irradiated with neutrons or proton to the levels required by the ITk outermost layer (NIEL $1.5 \times 10^{15} \mathrm{n}_{e q} / \mathrm{cm}^{2}$, TID $\left.80 \mathrm{Mrad}\right)$. Their efficiency and timing properties are tested in beam tests at either CERN SPS, PSI, ELSA, FNAL or DESY.

\subsection{Beamtest measurements on large electrode sensors}

As key performance parameter the hit efficiency was compared before and after neutron irradiation. The irradiations have been carried out the at the TRIGA reactor [13], Institute Jozef Stefan, Slovenia to $10^{15} \mathrm{n}_{e q} / \mathrm{cm}^{2}$. To measure hit efficency before and after irradiation, the sensors are placed in the center of a reference telescope, which reconstructs the particle's tracks and obtains a hit position prediction on the test sensor. The LF-Monopix sensor was tested at the ELSA accelerator [14], Bonn, Germany, using an EUDET-type reference telescope [15]. Figure 2 shows the hit efficiency as function of the track position before irradiation (a) and after irradiation (b). Integrated over the pixel area, the efficiency was found to be $99.6 \%$ before irradiation and $98.9 \%$ after irradiation [16].

The ATLASPix1Simple sensor was measured before and after irradiation in a beam test at the CERN SPS accelerator. The sensors were compared before and after $10^{15} \mathrm{n}_{e q} / \mathrm{cm}^{2}$ neutron irradiation. Using a ATLAS FE-I4 based telescope [17] and MuPix telescope [18], The global efficiency was measured across the matrix of the ATLASPix1Simple chip (400 rows and 25 columns) before 


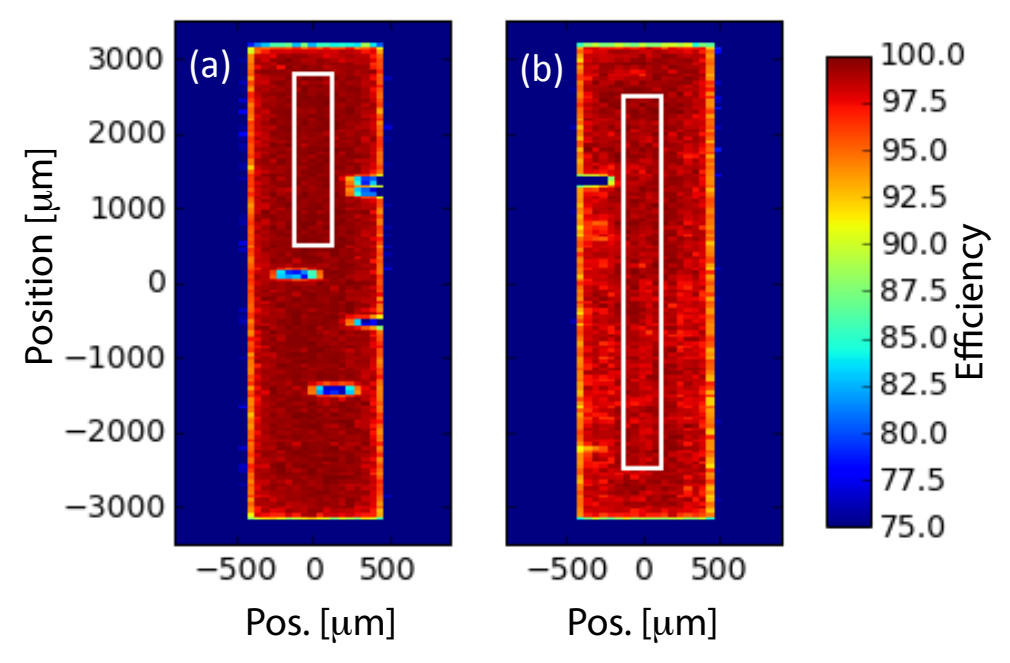

Figure 2: Hit efficiency of the LFMonoPix sensor before irradiation (a) and after $10^{15} \mathrm{n}_{e q} / \mathrm{cm}^{2}$ neutron irradiation (b) [16].

and after irradiation and is shown in figure 3. The measurements yield full efficiency of over $99 \%$ before and after irradiation without $25 \mathrm{~ns}$ time constraint. In order to determine the time-resolution the sensors were tested with the MuPix beam telescope [18] at DESY. Figure 4a shows the time resolution of the irradiated ATLASPix1Simple sensor with respect to the timing of the MuPix telescope.

The resolution of $15 \mathrm{~ns}$ includes the scintillator reference time and $16 \mathrm{~ns}$ binning of the system clock. The ATLASPix 1 time resolution includes the varying signal propagation delay along the column of around 50ns for the full column height. As the hit pixel position is known this propagation delay can be corrected for offline. Correcting for the propagation delay the sensor yields the time difference distribution shown in figure $4 \mathrm{~b}$ with $\sigma=9.8 \mathrm{~ns}$, which translates into a approximate in-time efficiency of $85 \%$ after irradiation.

\subsection{Measurements on small electrode sensors}

The MALTA and TJ-Monopix sensors implement a front-end circuit $[20,10]$ which is optimised for low-power operation of $75 \mathrm{~mW} / \mathrm{cm}^{2}$ while still sufficiently fast to be compatible with the $25 \mathrm{~ns}$ bunch structure of LHC. The small input capacitance of $<2 \mathrm{fF}$ allows one to achieve low noise operation $\left(\mathrm{ENC} \approx 15 \mathrm{e}^{-}\right)$at a constant front-end current of 500nA per pixel. For the MALTA sensor the matrix is readout through a novel asynchronous architecture, which is described in detail in reference [21]. The MALTA sensor also includes special monitoring pixels, which route the amplifier output signal to monitoring pads on the chip periphery without further digital circuit. This allows one to study the analog signal before the pixel descriminator. Figure 5a shows the unirradiated MALTA pixel analog pulse time-walk through the plot of time to threshold as function of analog amplitude in a test with a ${ }^{90} \mathrm{Sr}$ source. In this measurement only a single pixel is read out. As a results the plot also contains small signals with long time-walk, which stem from shared signals when an adjacent pixel is hit. To assess the in-time effiency the full sensor matrix is measured with 

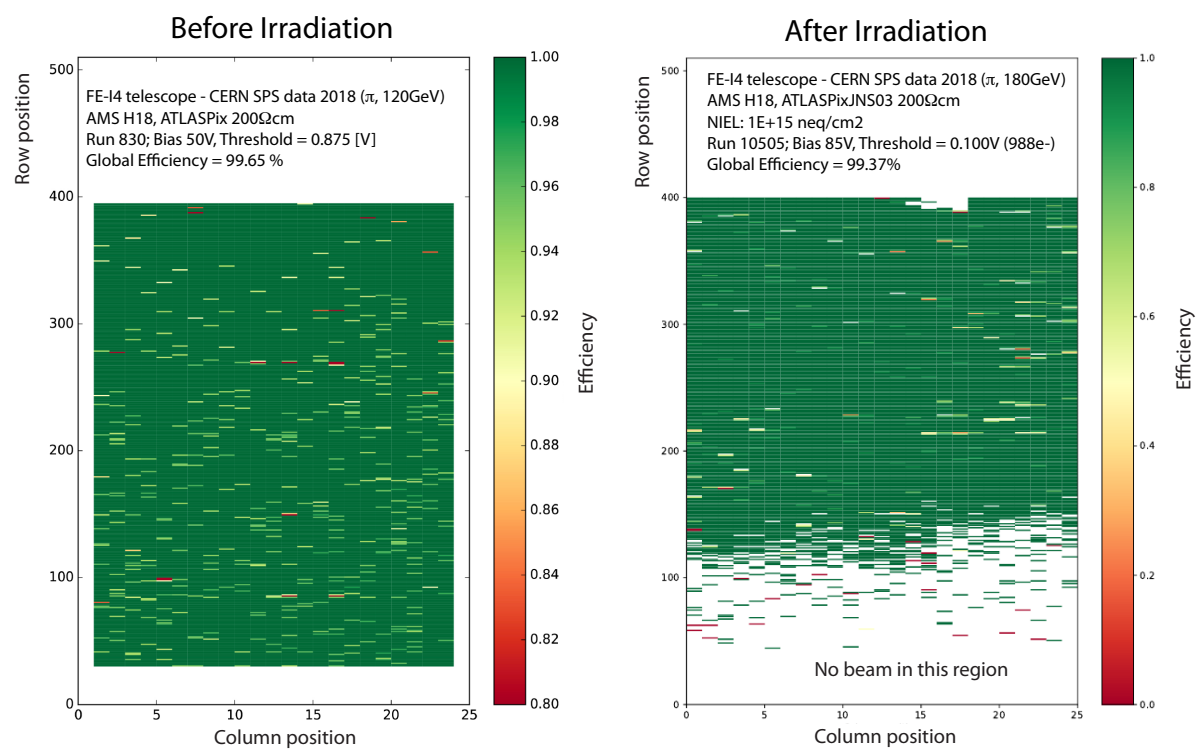

Figure 3: Hit efficiency of the ATLASPix1Simple sensor before irradiation (a) and after $10^{15} \mathrm{n}_{e q} / \mathrm{cm}^{2}$ neutron irradiation (b). [19]
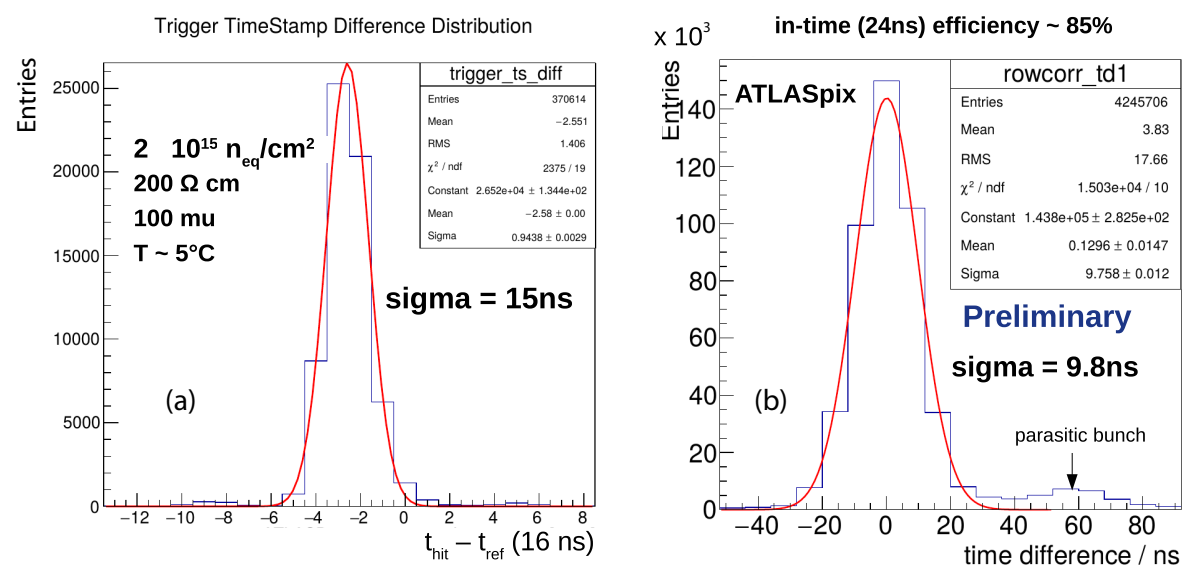

Figure 4: Time resolution of the ATLASPix1Simple sensor after $2 \times 10^{15} \mathrm{n}_{e q} / \mathrm{cm}^{2}$ neutron irradiation (a). Figure (b) shows the time-difference after unfolding the signal propagation delay in the sensor column.

${ }^{a}{ }^{90} \mathrm{Sr}$ source and a scintillator below the sensor. The scintillator provides the time reference. The arrival time of the MALTA signal is then measured on the chip periphery without unfolding the column propagation delay (max $7.5 \mathrm{~ns}$ for the full column height) with respect to the scintillator reference time. The resulting time-difference distribution is integrated in a sliding $25 \mathrm{~ns}$ window which is shown in figure $5 \mathrm{~b}$. The plot yields a in-time efficiency in $25 \mathrm{~ns}$ of $98 \%$.

The efficiency of the MALTA sensor has been studied before and after neutron irradiation in beam tests using a EUDET type telescope [15] at the CERN SPS accelerator. The MALTA 

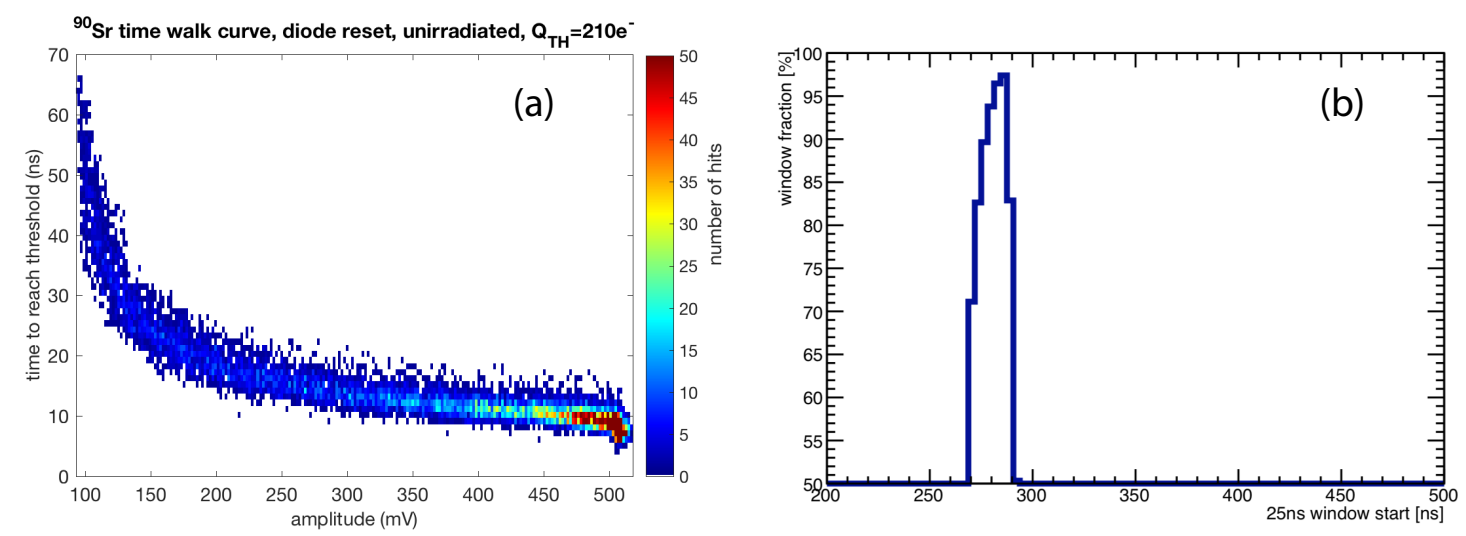

Figure 5: Analog pulse of the MALTA pixel (a). Figure (b) shows the integrated in-time efficiency in a $25 \mathrm{~ns}$ window as function of the window delay in a ${ }^{90} \mathrm{Sr}$ source test.

sensor was operated at room temperature for unirradiated and at $\approx-20^{\circ} \mathrm{C}$ for irradiated sensors. The MIMOSA planes of the telescope provided the track prediction to the sensor under test for the $180 \mathrm{GeV}$ pion beam. The precision of the telescope $(\approx 2 \mu \mathrm{m}[23])$ allows to study the efficency of the sensor as function of the hit position in the pixel. The matrix consists of different sectors using different deep p-well configurations $[20,21]$ under the p-well: in the standard configuration ("sector 2") the deep p-well extends all the way of the p-well to a $3.5 \mu$ m distance of the n-electrode . In a slightly modified configuration the deep p-well is retracted under the p-well as much as the placement of PMOS transistors allows (" reduced DPW sector 3"). The efficiency is studied also as function of the deep-p-well configuration. Figure 6a shows the in-pixel efficiency of the unirradiated sensor at an operational threshold of $250 \mathrm{e}^{-}$. The sensor shows full efficiency and we observed no dependence on the deep-p-well configuration. Figure $6 \mathrm{~b}$ shows the sensor after $10^{15} \mathrm{n}_{e q} / \mathrm{cm}^{2}$ neutron irradiation with a standard layout of deep p-well at a threshold of $350 \mathrm{e}^{-}$. The sensor exhibits good efficiency around the electrode however the efficiency drops sharply towards the pixel edge. The outline of the deep p-well is illustrated by the superimposed white rectangle at a $3.5 \mu \mathrm{m}$ distance of the n-electrode. In figure Figure $6 \mathrm{c}$ the same sensor shows higher efficiency in sector 3 when parts of the deep p-well are removed (white outlined polygone). The efficiency is improved towards the edges in the area where the deep p-well was removed.

This result leads to a modification of the implant structure to improve corner efficiency: Charge is lost in the pixel corner area due to the lack of a strong horizontal electrical field, which pushes the ionisation charge towards the electrode. Two changes have been implemented in the implant structure to achieve an increased horizontal field at the pixel boundary, which are shown in figure 7. In the modified process $[3,7]$ the full sensor surface includes a continuous $n$ - doped layer under the p-well structures. Figure (c) shows the horizontal electric field for this implant structure from pixel center to pixel center. In a first change an extra deep p-well ring around the pixel circumference has been added as shown in figure (a). Its resulting horizontal electric field is shown in figure (d) together with the electric field stream lines (black arrows). In a second change the n-layer is interrupted around the pixel circumference by a gap (figure 7b). The resulting horizontal field 


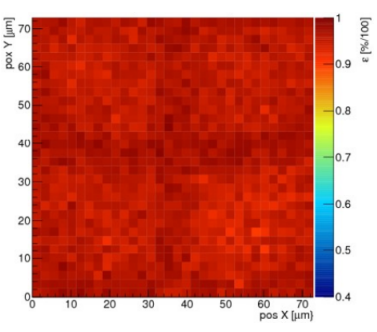

(a) Sector 2 unirradiated

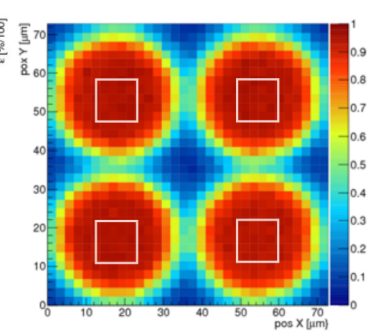

(b) Sector 2 neutron $1 \mathrm{E} 15 / \mathrm{cm}^{2}$

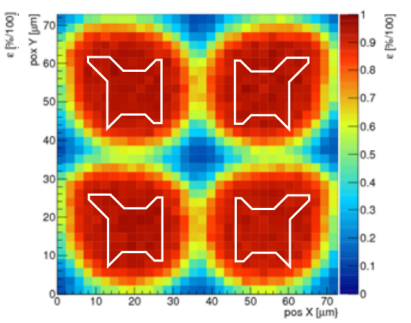

(c) Sector 3 neutron $1 \mathrm{E} 15 / \mathrm{cm}^{2}$

Figure 6: In-pixel efficiency of a $2 \times 2$ pixel array on an unirradiated MALTA sensor at a threshold of $250 \mathrm{e}^{-}$(a). Figure (b) shows the efficiency of the $10^{15} \mathrm{n}_{e q} / \mathrm{cm}^{2}$ sensor in sector 2 with standard deep-p-well configuration. Figure (c) shows the efficiency of sector 3 where the deep p-well has been partially removed under the p-well as outlined by the white polygone

and stream lines are shown in figure (e). The star symbol marks the location of the electric field minimum. Both changes result in stronger focusing of ionisation charges towards the collection electrode. Figure (d) and (e) show the significant increase in horizontal electric field due to this implant improvement which pushes the ionisation charge towards the collection electrodes.

The field configuration and transient current pulses are obtained in a 3D TCAD simulation of the pixel cell. Figure 8 shows the integrated charge as function of the integration time before irradiation (a) and after irradiation to $10^{15} \mathrm{n}_{e q} / \mathrm{cm}^{2}$ (b) [24]. The simulated incident particle is in the 4-pixel corner of 4 adjacent pixels, hence in the least favourable location for detection efficiency. The addition of an extra deep p-well (figure 7a) as well as a gap in the n- layer (figure 7b) results in significantly faster charge integration already before irradiation. After irradiation the relative improvement with these changes is even more substantial as the total integrated charge is a factor 2.5 to 3.5 higher with the new improvements. Based on these simulations the respective modifications to the implantation structures have been implemented in a new MALTA pixel matrix ("MiniMALTA"). The MiniMALTA sensor has been produced, irradiated and is currently under test with encouraging results.

\section{Summary}

Monolithic CMOS sensors present an interesting option as pixel detectors for the upgrade of tracking systems for the HL-LHC. Due to the absence of bump-bonding and two different dies for sensor and FE electronics, the detector assembly complexity and costs can be substantially reduced which is of great importance for future large pixel systems. In addition they hold the promise to improve performance through a reduction in pixel pitch and detector material. This paper summarizes the recent developments by the ATLAS CMOS pixel sensor collaboration for the development of first radiation hard monolithic CMOS sensors which can cope with the high radiation levels and substantial hit rates as expected in the future pixel trackers. We designed and tested several different sensors in $150 \mathrm{~nm}-180 \mathrm{~nm}$ CMOS imaging processes in view of radiation 


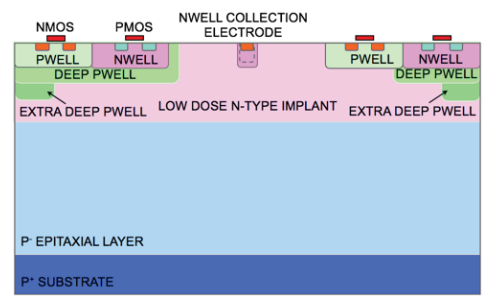

(a)

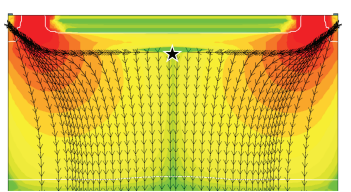

(c) Modified Process

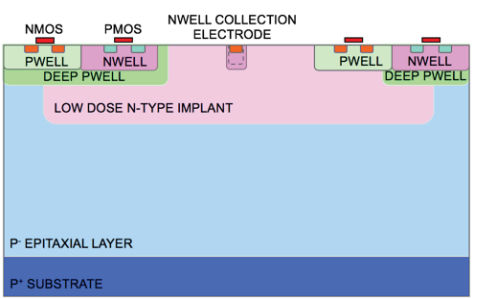

(b)

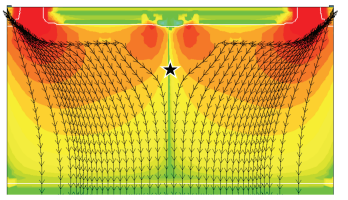

(e) With gap in $\mathrm{N}$ - layer

Figure 7: Changes to the implant structure to improve corner efficiency: (a) an extra deep p-well is added around the pixel circumference; (b) a gap in the n- layer is added around the circumference. A 3D TCAD simulation yields the horizontal electric field along the sensor depth (colour) and electric field stream lines (black arrows) for the modified process [3, 7] (c). Figure (d) shows the field configuration for the extra deep p-well as shown in (a), Figure (e) shows the field configuration for a gap in the n- layer as shown in (b).
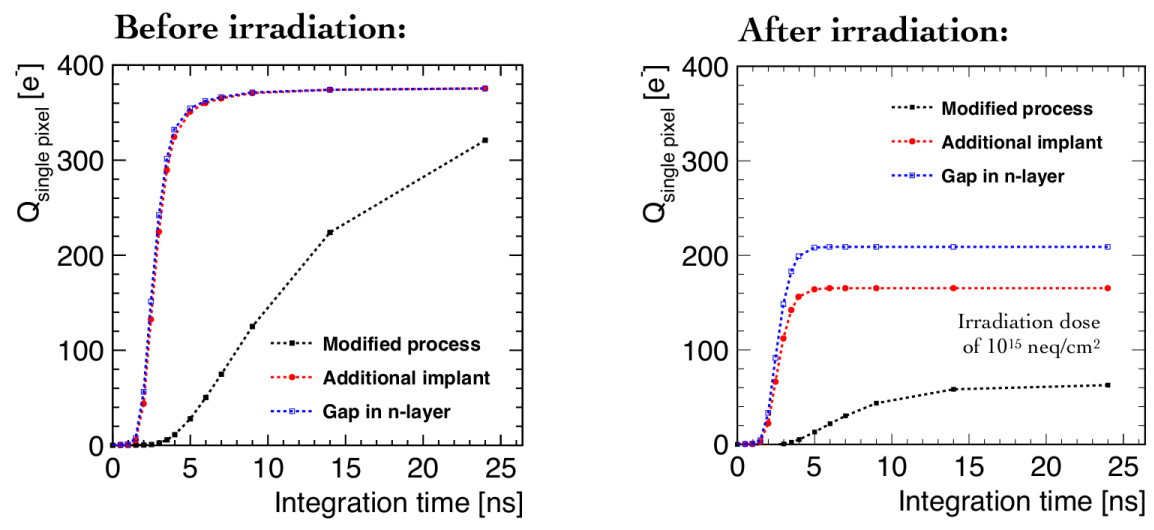

Figure 8: Integrated charge in 3D TCAD simulation as function of integration time before and after irradiation. The black curves shows the implant structure with continuous n- layer, the red curve shows the influence of an extra deep p-well and the blue curve shows the results of a gap in the nlayer. 
hardness and fast $25 \mathrm{~ns}$ signal response. The paper presents latest results on efficiency and timing response for so-called large electrode sensors and small electrode sensors.

\section{Acknowledgements}

The authors are grateful to Dr. Igor Mandic and Dr. Vladimir Cindro of the Institute Jozef Stefan, Ljubljana, Slovenia for their support during neutron irradiation campaign. The irradiation campaign has been supported by the H2020 project AIDA-2020, GA no. 654168. This research project has in part been supported by a Marie Sklodowska-Curie Innovative Training Network of the European Commission Horizon 2020 Programme under contract number 675587, STREAM.

\section{References}

[1] The ATLAS Collaboration, "The ATLAS Experiment at the CERN Large Hadron Collider", JINST 3 2008 S08003.

[2] The ATLAS Collaboration, "ATLAS Phase-II Upgrade Scoping Document”, CERN-LHCC-2015-020, http://cds.cern.ch/record/2055248

[3] W. Snoeys, "Monolithic CMOS sensors for high energy physics," Nucl. Instrum. Meth. A Volume 924 (2018) 51-58. doi:10.1016/j.nima.2018.06.034

[4] T. Kugathasan, "Review on CMOS sensors", these proceedings

[5] T. Wang et al.,"Development of a Depleted Monolithic CMOS Sensor in a $150 \mathrm{~nm}$ CMOS Technology for the ATLAS Inner Tracker Upgrade", 2017 JINST 12 C01039

[6] W. Snoeys et al., "A process modification for CMOS Monolithic Active Pixel Sensors for enhanced depletion, timing performance and radiation tolerance", Nucl. Instr. Meth. A Volume 871, 1 November 2017, pp. 90-96 doi:10.1016/j.nima.2017.07.046

[7] H. Pernegger et al., "First tests of a novel radiation hard CMOS sensor process for Depleted Monolithic Active Pixel Sensors", JINST 122017 P06008, http://iopscience.iop.org/article/10.1088/1748-0221/12/06/P06008

[8] T. Hemperek, "Overview and perspectives of depleted CMOS sensors for high radiation environments", PoS Vertex 2017 (2018) 034. doi:10.22323/1.309.0034

[9] I. Berdalovic et al., "Monolithic pixel development in TowerJazz $180 \mathrm{~nm}$ CMOS for the outer pixel layers in the ATLAS experiment", JINST 13 (2018) no.01, C01023. doi:10.1088/1748-0221/13/01/C01023

[10] K. Moustakas et al., "CMOS Monolithic Pixel Sensors based on the Column-Drain Architecture for the HL-LHC Upgrade”, Nucl. Instrum. Meth. (2018) doi:10.1016/j.nima.2018.09.100 [arXiv: 1809.03434 [physics.ins-det]].

[11] I. Mandič, V. Cindro, A. Gori?ek, B. Hiti, G. Kramberger, M. Zavrtanik, M. Miku? and T. Hemperek, "Charge collection properties of irradiated depleted CMOS pixel test structures", Nucl. Instrum. Meth. A 903 (2018) 126 doi:10.1016/j.nima.2018.06.062

[12] M. Kiehn et al., "Performance of CMOS pixel sensor prototypes in ams H35 and aH18 technology for the ATLAS ITk upgrade", doi:10.1016/j.nima.2018.07.061 arXiv:1807.05953 [physics.ins-det]. 
[13] L. Snoj, G. Azerovnik, A. Trkov, "Computational analysis of irradiationfacilities at the JSI TRIGA reactor”, Applied Radiation and Isotopes 70 (3)(2012) 483 Đ 488 doi:10.1016/j.apradiso.2011.11.042.

[14] N. Heurich, et al., "The new external beamline for detector tests at ELSA", Proc. of IPACÕ16, no. 7, 2016, pp. 4088 Đ 4090.doi:10.18429/JACoW-IPAC2016-THPOY00s2

[15] H. Jansen, et al., "Performance of the EUDET-type beam telescopes", EPJTechniques and Instrumentation 3 (1) (2016) 7.doi:10.1140/epjti/s40485-016-0033-2.

[16] T. Hirono et al., "Depleted Fully Monolithic Active CMOS Pixel Sensors (DMAPS) in High Resistivity 150 nm Technology for LHC”, Nucl. Instrum. Meth. A 924 (2019) 87-91

[17] M. Benoit et al., “The FE-I4 Telescope for particle tracking in testbeam experiments," JINST 11 (2016) no.07, P07003 doi:10.1088/1748-0221/11/07/P07003

[18] H. Augustin et al., “The MuPix Telescope: A Thin, high Rate Tracking Telescope,” JINST 12 (2017) no.01, C01087 doi:10.1088/1748-0221/12/01/C01087

[19] M. Kiehn et al., "Performance of the ATLASPix1 pixel sensor prototype in ams aH18 CMOS technology for the ATLAS ITk upgrade", Proceedings 9th Workshop on Semiconductor Pixel Detectors for Particles and Imaging (PIXEL) December 2018, Taipei, submitted to JINST

[20] I. Berdalovic et al., "MALTA: a CMOS pixel sensor with asynchronous readout for the ATLAS High-Luminosity upgrade", Proceedings of 2018 IEEE Nuclear Science Symposium and Medical Imaging Conference, in Press.

[21] R. Cardella et al., "MALTA: an asynchronous readout CMOS monolithic pixel detector for the ATLAS High-Luminosity upgrade.” Proceedings 9th Workshop on Semiconductor Pixel Detectors for Particles and Imaging (PIXEL) December 2018, Taipei, submitted to JINST

[22] M. Benoit [CLICdp Collaboration], "Pixel detector R\&D for the Compact Linear Collider," arXiv:1902.08752 [physics.ins-det].

[23] G. McGoldrick, Synchronized analysis of test beam data with the Judith software, Nucl. Instr. Meth. A Volume 765, 21 November 2014, pp. 140- 145

[24] M. Munker et al., "Simulations of CMOS sensors with a small collection electrode, improved for a faster charge collection and increased radiation tolerance", Proceedings 9th Workshop on Semiconductor Pixel Detectors for Particles and Imaging (PIXEL) December 2018, Taipei, submitted to JINST, arXiv:1903.10190 [physics.ins-det]. 